\title{
An Error Correction Methodology for Time Dependent Ontologies
}

\author{
Brett Drury, J.J. Almeida, and M.H.M. Morais \\ ${ }^{1}$ LIAAD-INESC \\ ${ }^{2}$ University of Minho \\ ${ }^{3}$ Department of Physics, I3N, University of Aveiro
}

\begin{abstract}
An increasing number of applications have become dependent upon information described in ontologies. Information may be correct for a limited period of time, for example, the assertion: "Barack Obama is the current president of the USA" will be incorrect in 2017. A presidential lifespan can be measured in years, however in a more dynamic domain, assertions may have lifespans of: months, weeks or days. In addition, erroneous relations may be introduced into an Ontology through mistakes in the information source or construction methodology. Ontologies which contain a large number of errors may impair the effectiveness of applications which depend on it. This paper describes an error correction methodology for ontologies automatically generated from news stories. The information contained in news stories can have a very limited lifespan, consequently constructing an Ontology by an addition of assertions will overtime accumulate errors. The proposed method avoids this problem through an assignment of a lifespan to each relation. A relation's lifespan is dependent upon: frequency of assertion, relation volatility and domain volatility. Once a relation's lifespan has elapsed the relation is either deleted or archived as a temporal "snapshot" of the domain. Individuals with 0 relations are also removed or archived. An evaluation of an Ontology constructed with the proposed scheme revealed a gain in the total number of relations overtime without an increase in the number of the errors. A comparison with an Ontology constructed with an accumulative addition of relations over an eight week period revealed that the proposed method reduced the error count by $81 \%$.
\end{abstract}

Keywords: Business News, Temporal Ontologies, Ontology Management.

\section{Introduction}

An increasing number of applications have become dependent upon information described in ontologies, for example news recommendation 1 and question answering 2. Ontologies which contain errors, ambiguous or "stale" information may impair the effectiveness of the applications which depend upon it. This problem is exacerbated by volatile domains because information changes quickly and consequently Ontologies which represent this area can quickly accumulate a large number of errors [3].

C. Salinesi and O. Pastor (Eds.): CAiSE 2011 Workshops, LNBIP 83, pp. 501 512, 2011.

(C) Springer-Verlag Berlin Heidelberg 2011 
The proposed method is an error correction methodology for Ontologies generated from news stories. News stories can provide a detailed and timely description of a specific area, however the information published in news is limited, either by the free market [4] or by public interest [5]. For example, an appointment of a new CEO may be published where as his retirement may not be newsworthy. The fallible nature of news ensures that ontologies generated from news information will contain errors [3] and current correction techniques [3] may not be optimal. The proposed method is predicated upon the redundancy of news information. The news publication process ensures that facts are repeated in multiple information sources and at regular intervals over time. The method assigns: 1. a nominal value (weight) to each relation, 2. a constant to represent the relation's volatility, 3. the last assertion date, 4 . a counter which represents the frequency of assertion. The nominal value is reduced overtime until a predefined value when the relation is deleted or archived. The relation's nominal value is refreshed with each new repetition and the decay of the nominal value is restarted. The speed of the decay of a relation's weight (lifespan) depends upon the following: 1. relation volatility, 2 . domain volatility and 3 . frequency of assertion. The combination of these factors ensures that a relation's lifespan may range from a single day to a number of years. The remainder of this paper will consider the following: 1 . initial Ontology construction, 2. adaptation of Ontology , 3. evaluation.

\section{Initial Ontology Construction}

\subsection{Centred Domain Ontology}

The central aim of this work is to construct a Centred Domain Ontology. A Centred Domain Ontology for the purposes of this paper is an Ontology constructed around an individual (an Ontology element). This research concentrated on economic actors: Companies, Government Institutions, Financial Markets as the central individual. The motivation was to represent in detail the domain of the economic actor. News was a natural medium because it contains up-to-date and detailed information about economic actors. The following section will describe an Ontology construction methodology which was used to construct an Ontology from news information [3] over a period of 53 days. The Ontology will be evaluated and it's failings will be illustrated.

\subsection{Ontology Construction}

The Ontology was constructed with the methodology described by Drury [3]. The construction process was separated into three parts: corpus construction, Ontology bootstrapping and enrichment.

Corpus construction. consisted of harvesting stories from free sources on the Internet. A crawler harvested these stories at the same time each day. The stories were published by the content providers in Really Simple Syndication (RSS) format. The crawler recorded Headline, Description, Story Text, HTML, Published Date and Harvested Date information. The content providers embedded 
links in the stories between related stories and background information. This information was recorded. Story text was extracted from the HTML and stored. The story text was sent to the Open Calais web service 6. The Open Calais web service provided meta-data for each story. This meta-data included relation extraction for the identified named entities in the story text.

Bootstrapping. the Ontology construction process involved identifying a highly relevant "seed set of news stories" from which relations can be extracted. The "seed set" was selected by searching the headlines for keywords. These stories were highly relevant because if a headline contained a keyword then the following story text would be relevant [7] to the aforementioned keyword. The relations were extracted from the Open Calais meta-data to construct the initial Ontology.

Ontology enrichment. was achieved by the expansion of the initial keywords with similar terms from the Ontology. The similar terms were identified by the OWL predicate "SameAS" 8] in the meta-data. This predicate referred to keywords which were the same as the initial keyword. In addition further information was gathered from Linked Data. The Linked Data was identified by following a unique URI provided by Open Calais for each named entity. The URI pointed to a "landing page" 1 on the Open Calais website. The "landing page" contained a "redirect" to the Linked Data web page from which extra relations can be extracted. The URI's which were redirected to the same Linked Data web page were considered to be instances of the same entity, consequently the URI provided a form of disambiguation. The construction and enrichment process is fully described in Algorithm 1, A simplified view of the Ontology generated with this method is demonstrated in Figure 2.2

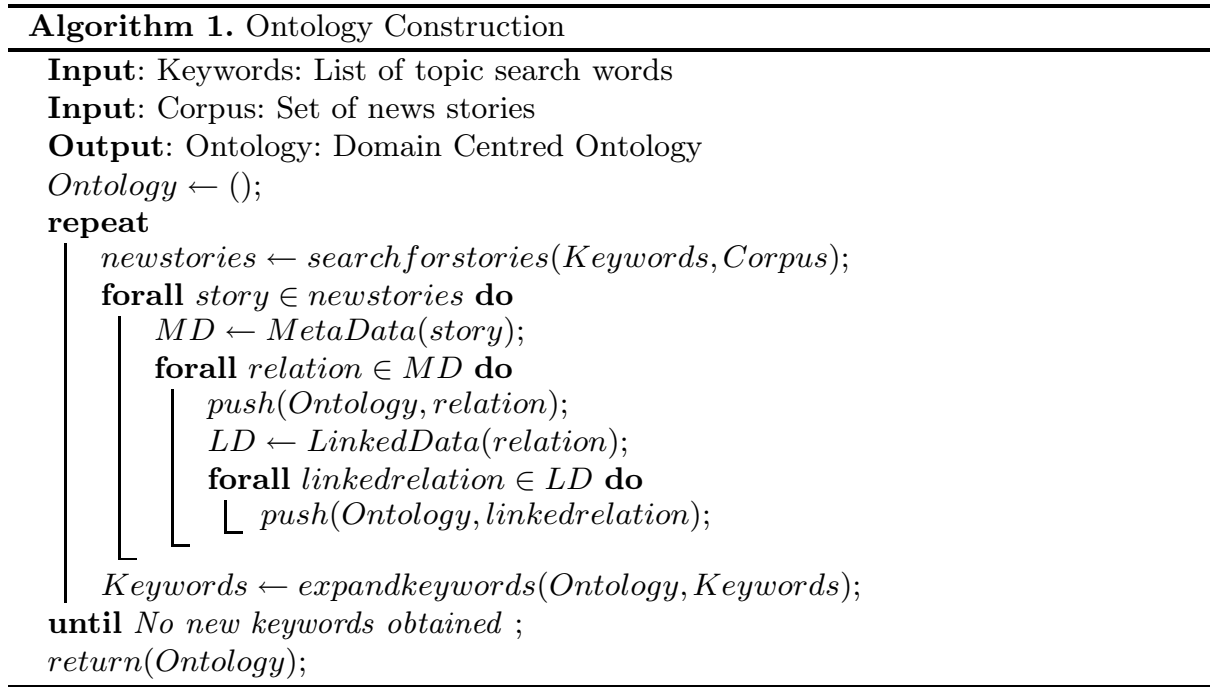

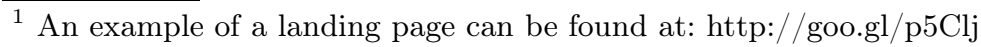




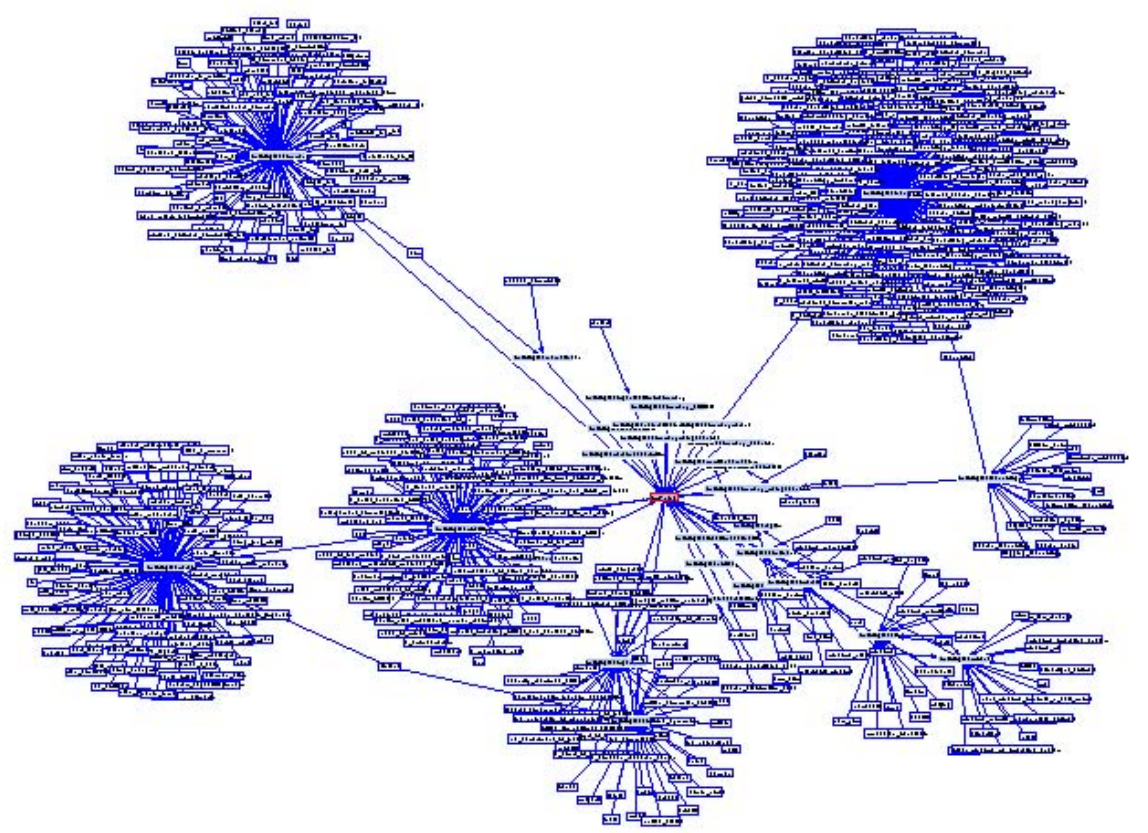

Fig. 1. Ontology viewed with Growl [3]

\subsection{Validation of Initial Ontology}

As previously described, an Ontology was generated from news stories over a period 53 days with the accumulative relation addition methodology [3]. This Ontology was to provide a baseline for comparison with the proposed method. The Ontology described the "Microsoft Domain". The Microsoft Domain was chosen to simplify the validation task because it was assumed that the main assertions should be "well known" or "easy" to verify. The Ontology validation was with Vrandecic's [9] tests. The Ontology was validated for: Accuracy, Adaptability, Clarity, Completeness, Computational efficiency, Conciseness, Consistency, and Organizational fitness. The validation was conducted manually by a domain expert. The Ontology was too large to validate in it's entirety, consequently the individual with the most relations was chosen for the validation tasks. The evaluation methodology obliged the evaluator to positively validate errors, consequently a relation was assumed to be "correct" if it could not be verified as incorrect. There was limited time to verify relations and consequently it was necessary to restrict the effort spent on verification. A positive error identification ensured that the manual verification task could be completed in the allocated time.

Accuracy: The individual with the most relations was the Microsoft individual. The Microsoft individual contained 436 relations. 61 relations were positively verified as incorrect. 
Clarity: The number of assertions were large, in-excess of 11,000. There were 10 class types and 20 relation types. Each class and relation type conformed to Vrandecic's 9 tests of understandability and transparency.

Completeness: The Microsoft individual relations were verified for completeness. There were a number of unverifiable relations, for example "Microsoft employs the Governor of California", however the verifiable relations provided a rich description of the domain. The assertions exceeded the experts knowledge and ability to locate all valid relations.

Conciseness: The Ontology contained a number of irrelevant axioms, for example "Microsoft employs current CEO"

Consistency: There were a number of axioms where were inconsistent, for example the Ontology asserted that both Microsoft and Adobe produced Acrobat Reader.

The initial Ontology failed three of Vrandecic's [9] validation tasks: conciseness, consistency and accuracy. A comparison of an Ontology generated with one day's information with one generated on day 53 indicated that the number of irrelevant and inconsistent axioms increased with time as did the correctly asserted axioms.

\section{Adaptation of Ontology}

The initial validation of the Ontology constructed with an accumulative relation addition methodology [3] indicated there were a significant number of positively verified errors. The errors could be categorized as: factual errors in the story text, errors in Open Calais meta-data, outdated information or irrelevant information in the story text. An absence of some form of error correction would imply that these errors would accumulate in numbers over time.

Drury 3] has attempted to use two error correction approaches to resolve this problem: 1. "contrary information",2. regular Ontology regeneration. "Contrary information" identifies contradictory relations. A relation which is not reasserted after the creation of the new contradictory relation is assumed to be false. Drury 3] provided the example of Jerry Yang and Carol Bartz, both were asserted to be the CEO of Yahoo!. After a certain period of time there were no reassertions for Jerry Yang as being the CEO of Yahoo!, but there were reassertions for Carol Bartz holding this position. Carol Bartz was correctly assumed to be the CEO of Yahoo. "Contrary information" is not a general error correction scheme, and requires manual tuning to identify the relations which can be corrected by this method. "Contrary information" also may be a non-optimal solution in the news domain because of the economics of news publication [4. The news publication process limits the amount of information published and consequently contradictory information may not be published. Regular regeneration of the Ontology may expel accumulated errors, but it will also remove correct relations. 
The proposed method seeks to avoid the disadvantages of the previously described error correction methodologies. As described earlier the proposed method is predicated upon the redundancy of news where "true" assertions are repeated in multiple locations and at regular intervals. The redundancy of information is created by the dynamics of news publication; the initial story is published by an agency [10 (Reuters,AP, UPI, AFP) and then is repeated by other news publishers. For example, the story concerning an appointment of a Microsoft executive to Nokia was repeated in our corpus by several information sources with slight differences in the headlines. The central assertion of the story was that "Stephen Elop is the CEO of Nokia", was repeated multiple times in each story and separately by multiple information sources [11] [12].

\subsection{Adaptation Measures}

The proposed decay of an assertion of a relation is influenced by three factors: 1 . domain volatility, 2. relation volatility and 3 . assertion frequency. The domain volatility, for the purposes of this paper, is the frequency of relation addition and repetition. The assumption is that the faster the addition and repetition of information the higher the rate of change of the information contained in the Ontology. A uniform relation decay rate may have ensured a too rapid relation deletion rate in static domains and a too slow deletion rate in dynamic domains. The domain volatility was calculated by counting the number of relevant stories over a fixed period of time. The relevant stories were selected by identifying domain keywords in the story headline. The assumption was that if a headline contained a domain keyword then the following story would be strongly relevant [7]. The relation volatility was a constant assigned by a domain expert. The assertion frequency was a count of the repetition of a relation assertion. The formulas are fully described below and the function which decays the relation weight is described in Algorithm 2 .

$$
\begin{aligned}
\text { DomVolatility } & =\frac{\text { NumberOfStories }}{\text { Constant }} \\
\text { TotalVolatility } & =\text { DomVolatility } \times \frac{\text { RelationVolatilityConstant }}{\text { NumberOfAssertions }} \\
\text { TotalLife } & =\frac{\text { MaximumLifeConstant }}{\text { TotalVolatility }} \\
\text { DecayRate } & =1-\frac{\text { DaysElapsed }}{\text { TotalLife }}
\end{aligned}
$$

\subsection{Decay Rate Characteristics}

There were three factors which directly influenced a lifespan of a relation: domain volatility, relation volatility and frequency of assertion. In addition the distribution of the reassertions over time, a property of the "frequency of assertion", also exerted an influence on the relation lifespan. This section will describe the 

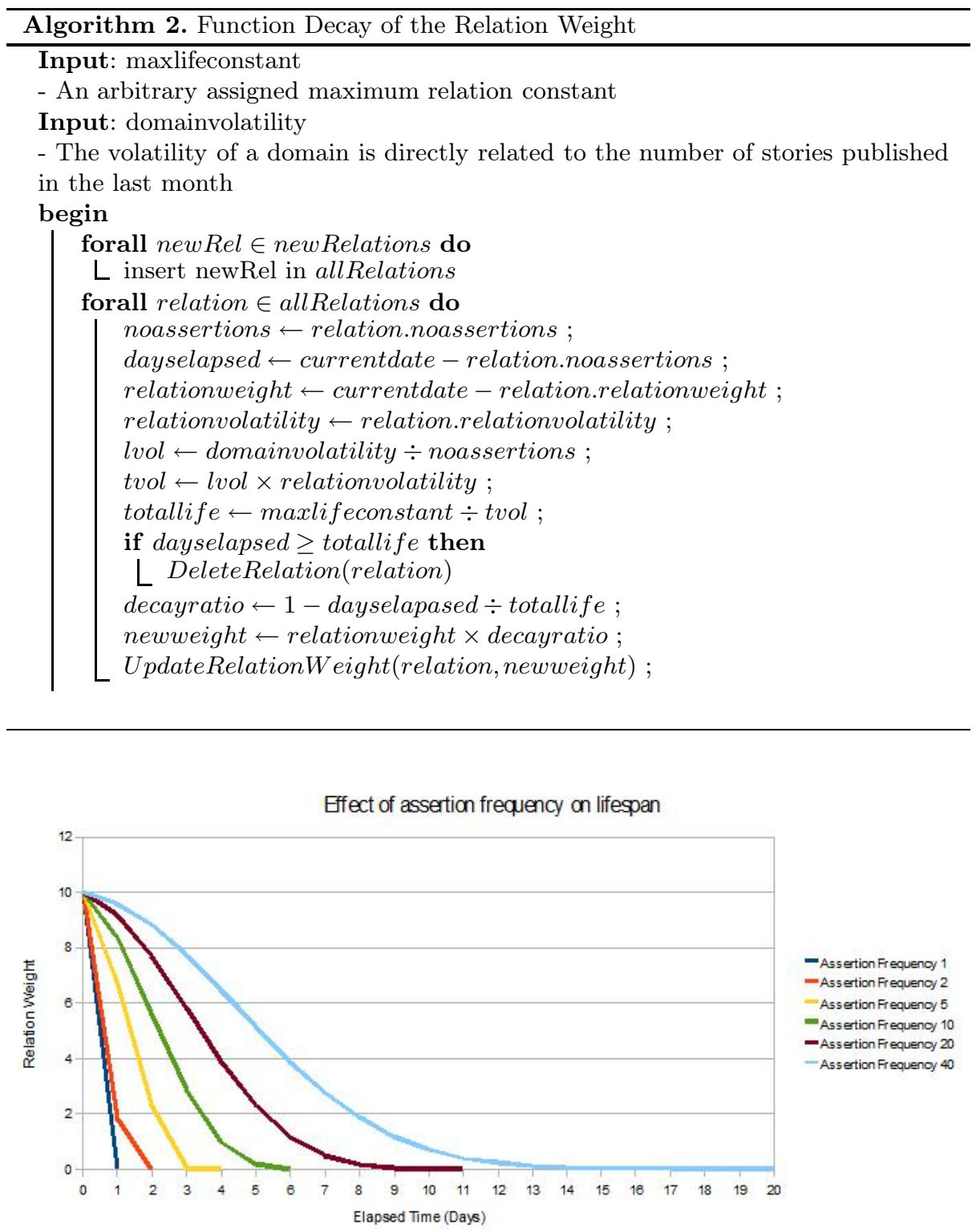

Fig. 2. Decay graph for relation with varying frequencies

effects of these factors on the decay rate on a relation of a fixed volatility. The influence of relation volatility has been excluded because of space considerations.

Assertion frequency: the influence on the decay rate is illustrated in figure 2. The data series, Assertion Frequency 1, is a relation which has been asserted once and consequently has a short lifespan which was 1 day. The data series, 


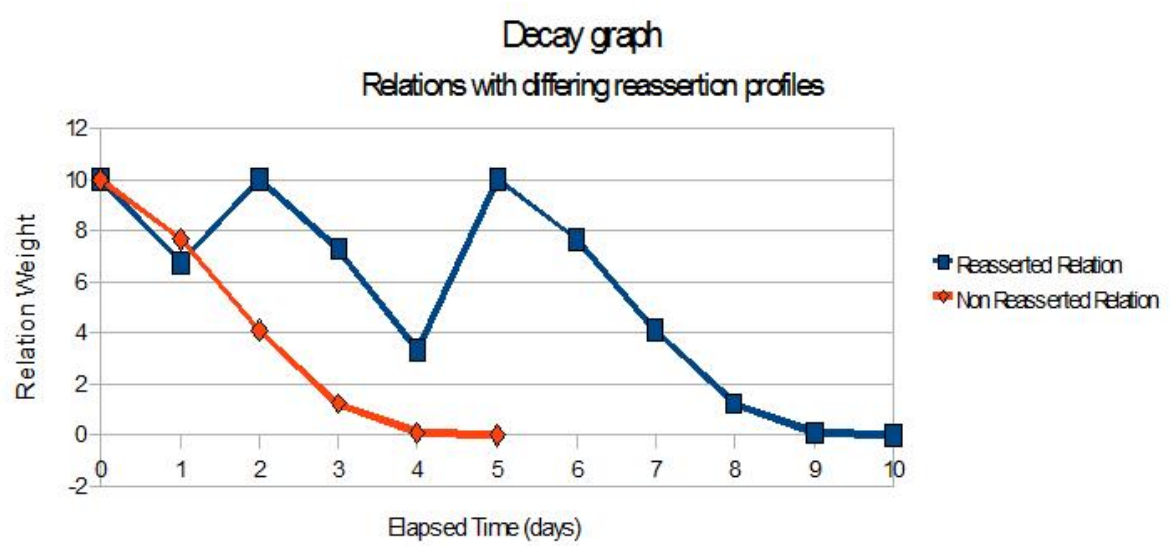

Fig. 3. Decay graphs for assertions with differing reassertions over time

Assertion Frequency 40, is a relation which has been asserted forty times and consequently has a longer lifespan which was 20 days.

Distribution of assertion frequency over time: has a direct effect on the relation's lifespan. The graph illustrated in Figure 3 demonstrates the effect of assertions over time. Each relation has an equal number of total assertions, however the data series: Reasserted relation, is reasserted at day 2 and day 5 . The reasserted relation has a longer lifespan than the non reasserted relation although both series have an equal number of total reassertions.

Domain volatility: has direct effect on a relation lifespan. The graph described in figure 4 illustrates the effect of lowering the domain volatility on a relation life. The data series, "domain volatility (standard)" has the domain volatility of the aforementioned Microsoft domain. The remaining data series represent a gradual decline in domain volatility from $80 \%$ of the Microsoft domain to $40 \%$. The decline of the volatility is represented by the labels "domain volatility (standard) -2 " to "domain volatility (standard) -6". Each label represents a decline in domain volatility of $20 \%$. The graph represents a clear increase in a relation's life as domain volatility declines.

\subsection{Decay Rate Proof}

This subsection is a brief discussion of the mathematical properties of the relation decay. It has been shown experimentally that the lifespan of a relation expands or contracts depending upon two factors (frequency of assertion and domain volatility). The larger the frequency of assertion and the smaller the domain volatility, the shallower the slope and consequently the larger life of the relation. The remainder of this section will show a mathematical proof that the characteristics shown on the graphs: Figure 2 and Figure 4 will continue for other values. 


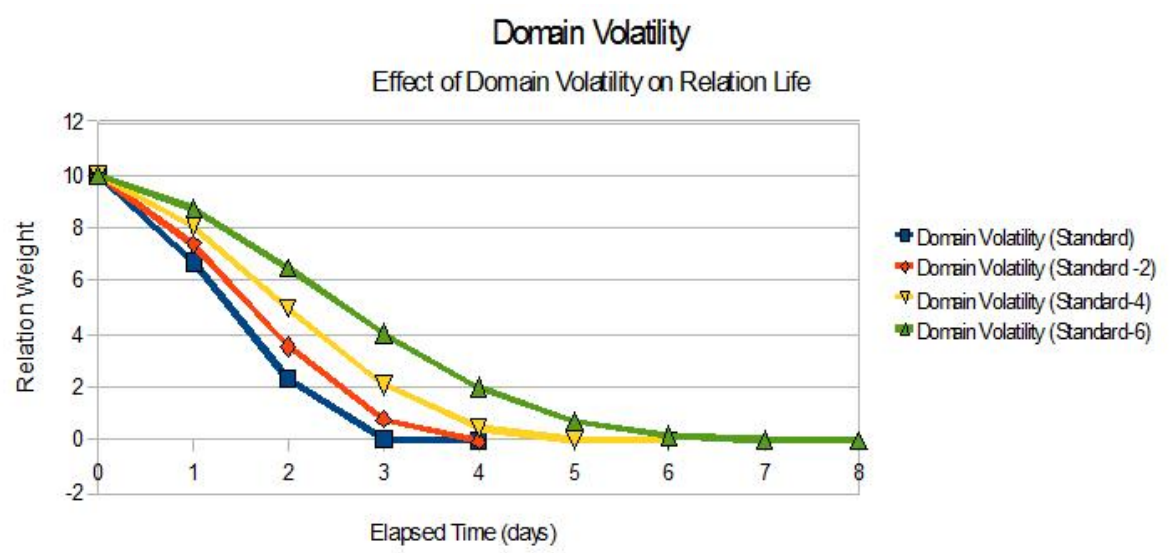

Fig. 4. Decay graphs for assertions with differing domain volatilities

\section{Gaussian Distribution Decay Proof}

The relation decay scheme (Figure 2 and Figure 4) follows approximately a Gaussian curve i.e. $y=y_{0} e^{-x^{2} / 2 \sigma^{2}}$. Therefore, the slope of the graph is shallower for larger standard deviation and this quantity can also provide a measure of the relation lifespan (when $x=3 \sigma$ we have $y=y_{0} / e^{4.5} \approx 0.01 y_{0}$ i.e. at time $3 \sigma$ the relation weight decreased to about $1 \%$ of the original value).

If $y=y_{0} e^{-x^{2} / 2 \sigma^{2}}$ then by the standard properties of the exponential and logarithm functions we have $\sqrt{-\ln \left(y / y_{0}\right)}=x /(\sqrt{2} \sigma)$. The values of $\sqrt{-\ln \left(y / y_{0}\right)}$ were plotted for each $x$ and we saw that the data fits straight lines with slope $1 /(\sqrt{2} \sigma)$. The values of the slopes and respective error bars obtained from the fits to the data in Figure 2 are $0.267 \pm 0.007$ (data set with Assertion Frequency 20 ) and $0.148 \pm 0.002$ (data set with Assertion Frequency 40). These values imply standard deviations $\sigma=2.65$ and $\sigma=4.77$, respectively, which as shown above, provide estimates of the relation lifespan. Therefore, it is possible to conclude that the relation decay scheme, when the assertion frequency is reasonably large, follows approximately a Gaussian curve. This implies that the larger the assertion frequency the longer the lifespan of the relation.

\section{Evaluation}

As described earlier, an Ontology was generated with the accumulation of relations from news methodology. This Ontology will be known as the "Original Ontology". For the evaluation, an Ontology which was generated with the proposed method will be known as the "Modified Ontology". The evaluation of the proposed methodology was by a comparison of the Modified Ontology with the Original Ontology. The Ontologies were generated from news gathered from a period of 53 days. Intermediate Ontologies were archived on a daily basis. A 


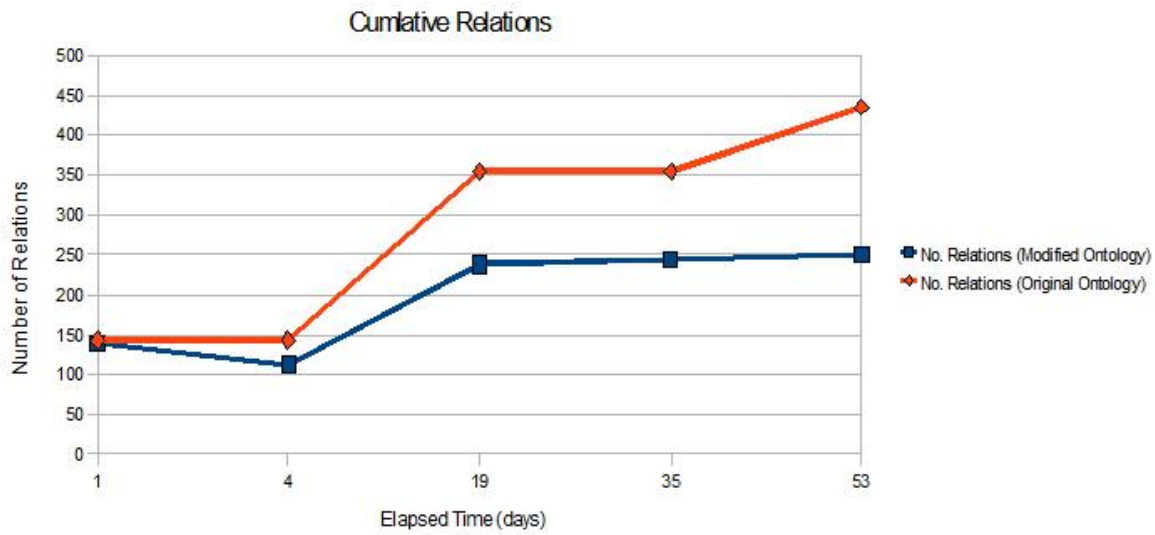

Fig. 5. Cumulative number of relations

random selection of the intermediate Ontologies were evaluated. The manual evaluation used the same methodology as the initial validation: 1 . the individual with most relations was evaluated, 2 . errors had to be positively identified and 3 . relations which were not positively validated as false were assumed to be correct.

Results. The cumulative number of errors is presented in Figure 6. As expected, the Original Ontology gains errors overtime, where as the Modified Ontology has an initial reduction in the total number of errors and thereafter the number of errors is almost constant over time. The total number of relations for both Ontologies is described in Figure 5. The Original Ontology gained more relations than the Modified Ontology, but the error rate is significantly less for the Modified Ontology. The figures for total number of errors and the error rate are presented in the following table.

\begin{tabular}{|l|l|l|l|l|}
\hline Days Elapsed & $\begin{array}{l}\text { Error Rate } \\
\text { (M.O.) }\end{array}$ & $\begin{array}{l}\text { Error Rate } \\
\text { (O.O.) }\end{array}$ & $\begin{array}{l}\text { No. Errors } \\
\text { (M.O.) }\end{array}$ & $\begin{array}{l}\text { No. Errors } \\
\text { (O.O.) }\end{array}$ \\
\hline 1 & $11 \%$ & $13 \%$ & 15 & 18 \\
\hline 4 & $4 \%$ & $13 \%$ & 5 & 18 \\
\hline 19 & $4 \%$ & $10 \%$ & 10 & 37 \\
\hline 35 & $6 \%$ & $10 \%$ & 14 & 37 \\
\hline 53 & $5 \%$ & $14 \%$ & 12 & 63 \\
\hline
\end{tabular}

Comparison of error rates and number of relations

O.O. $=$ Original Ontology, M.O. $=$ Modified Ontology

\subsection{Discussion of Results}

The evaluation criteria underplayed the effectiveness the proposed methodology because: 1 . errors had to be "positively" identified, 2. the "quality" of the 


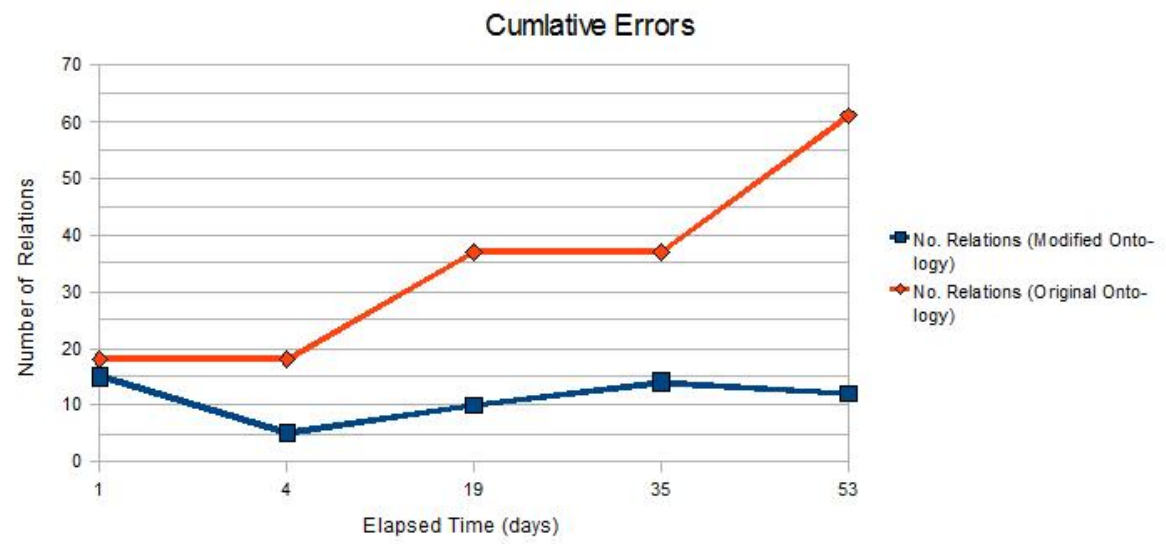

Fig. 6. Cumulative number of errors

relations were not considered. There were a larger number of "unverifiable relations" in the Original Ontology than the Modified Ontology. The "unverifiable relations" were not "obviously wrong", however it was not clear if they were part of the domain. The "unverifiable relations" accounted for the significant part of the difference in total relations between the Modified and Original Ontology.

The evaluation criteria did not consider the ability of the Modified Ontology to resolve ambiguous relations, for example at day 19 both Ontologies asserted that Microsoft and Adobe produced Acrobat Reader. In the Modified Ontology there was a measure of "confidence" for both relations in a form of a "relation weight". The "relation weight" for the assertion "Adobe produces Acrobat Reader" was higher than the weight for the assertion "Microsoft produces Acrobat Reader". It was possible to identify the "true" relation by accepting the relation with the highest weight.

It was not possible to evaluate the "completeness" of the Ontology as it was not feasible to generate a "Gold Standard" of all of the relations and individuals in the domain, however the Modified Ontology contained a number of stable relations which described the commonly known "facts" of the domain. The stable relations did not exit the Ontology at the points the intermediate ontologies were manually evaluated.

\section{Conclusion and Future Work}

The proposed methodology provides a basis for an automatic construction of a "timely" Ontology from news sources. The correction scheme removes: erroneous and "stale" or inconsequential information. The method also provides a procedure for resolving ambiguous relations via a "relation weight". The Modified Ontology formed the basis of a "breaking financial news" recommender system which ranked news stories in order of "importance" to a domain. An evaluation 
of the recommender system demonstrated a clear advantage for the system which used this methodology [13].

There were some weaknesses in the proposed technique. A number of values in the decay formulas were arbitrary. It was assumed that the relationships of these values were correct, but the relationships may be non-optimal. The future work will be to discover the optimal rates for these values. It may be possible to use machine learning $(\mathrm{ml})$ to discover the optimal relationship between relation addition and expulsion. As stated earlier, it was not possible to produce a "Gold Standard" of domain relations, however it is possible to produce a "Gold Standard" of "breaking news stories". The relative performance of competing Ontologies can measured by the "distance" between the news stories recommended by a news recommender which relies upon the Ontology. A learner may be able to identify optimal values for the decay formulas.

\section{References}

1. Flavius, F., Borsje, J., Levering, L., Embregts, H., Frasincar, F.: Hermes: an ontology-based news personalization portal (2007)

2. Lopez, V., Uren, V., Motta, E., Pasin, M.: Aqualog: An ontology-driven question answering system as an interface to the semantic web. Journal of Web Semantics 5(2), 72-105 (2007)

3. Drury, B., Almeida, J.J.: Construction of a local domain ontology from news stories. In: Lopes, L.S., Lau, N., Mariano, P., Rocha, L.M. (eds.) EPIA 2009. LNCS, vol. 5816, pp. 400-410. Springer, Heidelberg (2009)

4. McManus, J.: An economic theory of news selection. In: Annual Meeting for Education in Journalism and Mass Communication (1988)

5. Rosengren, K.E.: International news: Methods, data and theory. Journal of Peace Research 11(2), 145-156 (1974)

6. Reuters. Calais web service, http://opencalais.com/ (consulted in 2009)

7. Blake, C.: Andrew. Media-generated shortcuts: Do newspaper headlines present another roadblock for low-information rationality? The Harvard International Journal of Press/Politics 12(2), 24-43 (2007)

8. McGuinness, D.L., van Harmelen, F., et al.: OWL Web Ontology Language Overview. W3C Recommendation 10, 2004-2003 (2004)

9. Ontology Evaluation. In: Staab, S., Studer, R. (eds.) Handbook on Ontologies. International Handbooks on Information Systems, pp. 293-313. Springer, Heidelberg (2004)

10. Bell, A.: The Language of News Media. Language in Society, Blackwell (1991)

11. BBC. Nokia appoints microsoft executive as new head, http://www.bbc.co.uk/ news/business-11257069 (consulted September 2010)

12. Yahoo. Nokia replaces ceo with microsoft exec in smart phone war, http://finance. yahoo. com/news/Nokia-Replaces-CEO-With-ibd- 4210132396. html? $=0 \&$. v=1 (consulted September 2010)

13. Drury, B., Almeida, J.J.: Magellan: An adaptive ontology driven "breaking financial news" recommender. In: CISTI Proceedings (2011) 